\title{
Approximation Algorithms for the Highway Problem under the Coupon Model*
}

\author{
Ryoso Hamane \\ hamane@dac.gsic.titech.ac.jp titoh@dac.gsic.titech.ac.jp tomita@dac.gsic.titech.ac.jp \\ Tokyo Institute of Technology
}

\begin{abstract}
When a store sells items to customers, the store wishes to decide the prices of items to maximize its profit. Intuitively, if the store sells the items with low (resp. high) prices, the customers buy more (resp. less) items, which provides less profit to the store. So it would be hard for the store to decide the prices of items. Assume that the store has a set $V$ of $n$ items and there is a set $E$ of $m$ customers who wish to buy the items, and also assume that each item $i \in V$ has the production cost $d_{i}$ and each customer $e_{j} \in E$ has the valuation $v_{j}$ on the bundle $e_{j} \subseteq V$ of items. When the store sells an item $i \in V$ at the price $r_{i}$, the profit for the item $i$ is $p_{i}=r_{i}-d_{i}$. The goal of the store is to decide the price of each item to maximize its total profit. We refer to this maximization problem as the item pricing problem. In most of the previous works, the item pricing problem was considered under the assumption that $p_{i} \geq 0$ for each $i \in V$, however, Balcan, et al. [In Proc. of WINE, LNCS 4858, 2007] introduced the notion of "loss-leader," and showed that the seller can get more total profit in the case that $p_{i}<0$ is allowed than in the case that $p_{i}<0$ is not allowed. In this paper, we consider the line highway problem (in which each customer is interested in an interval on the line of the items) and the cycle highway problem (in which each customer is interested in an interval on the cycle of the items), and show approximation algorithms for the line highway problem and the cycle highway problem in which the smallest valuation is $s$ and the largest valuation is $\ell$ (this is called an $[s, \ell]$-valuation setting) or all valuations are identical (this is called a single valuation setting).
\end{abstract}

Keywords: Line Highway Problem, Cycle Highway Problem, Multi-Valuations, Single-Valuation.

\section{Introduction}

\section{$1.1 \quad$ Background}

When a store sells items to customers, the store wishes to decide the prices of items to maximize its profit. Intuitively, if the store sells the items with low (resp. high) prices, then the customers buy more (resp. less) items, which provides less profit to the store. So it would be hard for the store to decide the prices of items. Assume that the store has a set $I=\{1,2, \ldots, n\}$ of $n$ items and there is a set $C=\left\{c_{1}, c_{2}, \ldots, c_{m}\right\}$ of $m$ customers who wish to buy the items. The goal of the store is to decide the price of each item to maximize its profit. We refer to this problem as the item pricing problem. We classify the item pricing problem according to how many items the store can sell and how the customers valuate items. If the store can sell each item $i$ with unlimited (resp. limited) amount, we refer to this as the unlimited (resp. limited) supply model. The item pricing problem is said to be single-minded [10] if each customer $c_{j} \in C$ is interested in only a single bundle $e_{j}=\left\{j_{1}, j_{2}, \ldots,\right\} \subseteq I$ of items with valuation $v_{j} \geq 0$ and has valuation " 0 " on all other bundles of items. We say that the item pricing problem is unit-demand [10] if each customer $c_{j} \in C$ assigns valuation $v_{j}^{i} \geq 0$ to each item $i \in I$ and buys one of the most beneficial items for $c_{j} \in C$.

By regarding the set $I$ of $n$ items as the set $V$ of $n$ vertices and the set $C$ of $m$ customers as the set $E$ of $m$ hyperedges, each of which has weight $v_{j} \geq 0$, this can be formulated by a weighted hypergraph $\tilde{G}=\left(V, E,\left\{v_{j}\right\}\right)$. Note that the hypergraph $\tilde{G}$ might have selfloops (corresponding to customers that are interested in a single item) and multiedges (corresponding to customers that want to get the same bundle of items). For a weighted hypergraph $\tilde{G}=\left(V, E,\left\{v_{j}\right\}\right)$, assume that each item $i \in V$ has the production cost $d_{i}$ and each customer $e_{j} \in E$ has the valuation $v_{j}$. For $\tilde{G}$, we define a reduced instance $G=\left(V, E,\left\{w_{j}\right\}\right)$ to

\footnotetext{
* This research was supported in part by JSPS Global COE program "Computationism as a Foundation for the Sciences."
} 
be $w_{j}=v_{j}-\sum_{i \in e_{j}} d_{i}$ for each $e_{j} \in E$. If an item $i \in V$ is assigned a price $p_{i}$ in the reduced instance $G$, then its selling price is given by $r_{i}=p_{i}+d_{i}$. In this paper, we focus on the single-minded and unlimited supply model and consider reduced instances $G$ 's of weighted hypergraphs. We say that $G=\left(V, E,\left\{w_{j}\right\}\right)$ is an instance of the $k$-hypergraph vertex pricing problem if $\left|e_{j}\right| \leq k$ for each $e_{j} \in E$, an instance of the graph vertex pricing problem if $\left|e_{j}\right| \leq 2$ for each $e_{j} \in E$, and an instance of the bipartite graph vertex pricing problem if $G$ is a bipartite graph. As a special case of the hypergraph vertex pricing problem, we also say that $G=$ $\left(V, E,\left\{w_{j}\right\}\right)$ is an instance of the highway problem if each $e_{j} \in E$ is an interval on $V$ (the definition will be given in Definition 2.5 for the line highway problem and in Definition 2.6 for the cycle highway problem).

In most of the previous works [1, 4, 5, 10, the item pricing problem is considered under the model that $p_{i} \geq 0$ for each item $i \in V$ (this is called the positive price model). By introducing the notion of loss-leader [6], however, Balcan, et al. [3] consider several price models in which $p_{i}<0$ for some item $i \in V$ (these are referred to as the discount model, the B-bounded discount model, the coupon model, etc., and are formally defined in Subsection 2.1), and showed that the seller could get more profit in the case that $p_{i}<0$ is allowed than in the case that $p_{i}<0$ is not allowed.

\subsection{Related Works}

\subsubsection{Positive Price Models}

For the hypergraph vertex pricing problem, Guruswami, et al. [10, Theorem 5.2] show an $O(\log m+\log n)$ approximation algorithm. On the other hand, Demaine, et al. [7, Theorem 3.2] present that it is hard to approximate the hypergraph vertex pricing problem within a factor of $\log ^{\delta} n$ for some $\delta>0$ under the assumption that $\mathrm{NP} \nsubseteq \operatorname{BPTIME}\left(2^{n^{\epsilon}}\right)$ for some $\epsilon>0$. For the $k$-hypergraph vertex pricing problem, Briest and Krysta [4, Theorem 5.1] show an $O\left(k^{2}\right)$-approximation algorithm, which is improved to an $O(k)$-approximation algorithm [1, Theorem 2]. For the graph vertex pricing problem, Balcan and Blum derive a 1/4-approximation algorithm [1, Theorem 1], while by the reduction from the vertex cover, Guruswami, et al. 10, Theorem 3.1] show that the graph vertex pricing problem is APX-hard even when all valuations are identical (if selfloops are allowed) or all valuations are either 1 or 2 (if selfloops are not allowed). For the highway problem, Balcan and Blum [1, Theorem 3] show an $O(\log n)$-approximation algorithm and for the highway problem that forms a hierarchy, Balcan and Blum [1, Theorem 4] show a fully polynomial time approximation scheme. For the nonapproximability for the highway problem, see [4, 9].

\subsubsection{Other Models Based on Loss-Leader}

For the highway problem, we know the $\Omega(\log n)$ gap between the positive price model and the ( $B$-bounded) discount model [2, Theorem 1], and the $\Omega(\log n)$ gap between the coupon model and the $(B$-bounded) discount model [2, Theorem 2]. For the graph vertex pricing problem, the $\Omega(\log n)$ gap between the positive price model and the $B$-bounded discount model [2, Theorem 3] is known. For the highway problem, Balcan, et al. [3, Theorem 3] show a 2.33-approximation algorithm under the coupon model if all valuations are identical and for the highway problem on tree, Balcan, et al. [2, Theorem 15] show a 4-approximation algorithm under the coupon model if all valuations are identical.

\subsection{Main Results}

In this paper, we consider the highway problem with $[s, \ell]$-valuation, which is the highway problem with the smallest valuation $s$ and the largest valuation $\ell$. We also classify the highway problem into the line highway problem and the cycle highway problem in which each interval is defined on the line of items and the cycle of items, respectively. Then we consider the line highway problem with $[s, \ell]$-valuation and the cycle highway problem with $[s, \ell]$-valuation and a single valuation. 
Theorem 3.3: On an instance $G=\left(V, E,\left\{w_{j}\right\}\right)$ of the line highway problem with $[s, \ell]$-valuation, the algorithm $\operatorname{LiNE}_{[s, \ell]}$ outputs a price vector $\boldsymbol{p}$ that satisfies

$$
\frac{\operatorname{Opt}_{\text {coup }}(G)}{\mathbf{E}\left[\operatorname{Profit}_{\text {coup }}(\boldsymbol{p})\right]} \leq\left\{\begin{array}{cl}
4(1-\ln r) & 0 \leq r \leq \alpha, 1 / \sqrt{e} \leq r \leq 1 \\
3 / r & \alpha<r \leq 1 / 2 \\
6 & 1 / 2<r<1 / \sqrt{e}
\end{array}\right.
$$

where $r=s / \ell$ is the ratio between the smallest and the largest valuations and $\alpha \approx 0.3824$, i.e., $\alpha$ is the solution of the equality $3 / x=4(1-\ln x)$.

Theorem 4.1: On an instance $G=\left(V, E,\left\{w_{j}\right\}\right)$ of the cycle highway problem with $[s, \ell]$-valuation, the algorithm $\mathrm{CYCLE}_{[s, \ell]}$ outputs a price vector $\boldsymbol{p}$ that satisfies

$$
\mathrm{OPT}_{\text {coup }}(G) \leq 4(1-\ln r) \cdot \mathbf{E}\left[\operatorname{PrOFIT}_{\text {coup }}(\boldsymbol{p})\right]
$$

where $r=s / \ell$ is the ratio between the smallest and the largest valuations and $\alpha \approx 0.3824$, i.e., $\alpha$ is the solution of the equality $3 / x=4(1-\ln x)$.

Theorem 4.2; On an instance $G=(V, E)$ of the cycle highway problem with a single valuation, the algorithm CYC_Single_VAL outputs a price vector $\boldsymbol{p}$ that satisfies

$$
\operatorname{OPT}_{\text {coup }}(G) \leq 2.747 \cdot \operatorname{PrOFIT}_{\text {coup }}(\boldsymbol{p}) .
$$

For the line highway problem, Theorem 3.3 is an extension of the 2.33-approximation algorithm with a single valuation due to Balcan, et al. [3, Theorem 3]. The cycle highway problem is introduced in this paper as a generalization of the line highway problem, and Theorem 4.2 can be regarded as an extension of the 2.33approximation algorithm for the line highway problem with a single valuation [3, Theorem 3].

\section{Preliminaries}

\subsection{Price Models}

Let $G=\left(V, E,\left\{w_{j}\right\}\right)$ be a reduced instance of the item pricing problem. For a hyperedge $e_{j} \in E$ and a price vector $\boldsymbol{p}=\left(p_{1}, p_{2}, \ldots, p_{n}\right)$ over the $n$ vertices, let $p\left(e_{j}\right)=\sum_{i \in e_{j}} p_{i}$ be the sum of the profit on $e_{j} \in E$, i.e., the profit that is returned from the customer $e_{j} \in E$ for the price vector $\boldsymbol{p}$.

In most of the previous works [1, 4, 5, 10, the item pricing problem is considered under the model that $p_{i} \geq 0$ for each item $i \in V$. By introducing the notion of loss-leader, however, Balcan, et al. [3] considered several price models in which $p_{i}<0$ for some item $i \in V$, and showed that the seller could get more profit in the case that $p_{i}<0$ is allowed than in the case that $p_{i}<0$ is not allowed. In the following, we formally present the definitions of price models [3] with respect to the reduced instance.

Definition 2.1 (Positive Price Model) Under the condition that $p_{i} \geq 0$ for each $i \in V$, find a price vector $\boldsymbol{p}=\left(p_{1}, p_{2}, \ldots, p_{n}\right)$ that maximizes $\operatorname{PROFIT}_{\mathrm{pos}}(\boldsymbol{p})=\sum_{e_{j} \in E: w_{j} \geq p\left(e_{j}\right)} p\left(e_{j}\right)$.

Definition 2.2 (Discount Model) Find a price vector $\boldsymbol{p}=\left(p_{1}, p_{2}, \ldots, p_{n}\right)$ that maximizes $\operatorname{PROFIT}_{\text {disc }}(\boldsymbol{p})=$ $\sum_{e_{j} \in E: w_{j} \geq p\left(e_{j}\right)} p\left(e_{j}\right)$.

Definition 2.3 ( $B$-Bounded Discount Model) Under the conditions that $d_{i}=B$ and $p_{i} \geq-B$ for each $i \in$ $V$, find a price vector $\boldsymbol{p}=\left(p_{1}, p_{2}, \ldots, p_{n}\right)$ that maximizes $\operatorname{PROFIT}_{B}(\boldsymbol{p})=\sum_{e_{j} \in E: w_{j} \geq p\left(e_{j}\right)} p\left(e_{j}\right)$.

Definition 2.4 (Coupon Model) Find a price vector $\boldsymbol{p}=\left(p_{1}, p_{2}, \ldots, p_{n}\right)$ that maximizes $\operatorname{PROFIT}_{\text {coup }}(\boldsymbol{p})=$ $\sum_{e_{j} \in E: w_{j} \geq p\left(e_{j}\right)} \max \left\{p\left(e_{j}\right), 0\right\}$.

Under the coupon model, if $w_{j} \leq 0$, then $e_{j}$ never contributes to the profit for any price vector $\boldsymbol{p}$. So without loss of generality, we assume that $w_{j}>0$ for each $j \in[1, m]$ under the coupon model. 


\subsection{Highway Problem}

For any pair of integers $a \leq b$, let $[a, b]=\{a, a+1, \ldots, b\}$. Informally, we say that $G=\left(V, E,\left\{w_{j}\right\}\right)$ is an instance of the line highway problem [10] if each $e_{j} \in E$ is an interval in the line on $V$. We introduce the cycle highway problem as a generalization of the line highway problem, and we say that $G=\left(V, E,\left\{w_{j}\right\}\right)$ is an instance of the cycle highway problem if each $e_{j} \in E$ is an interval in the cycle on $V$.

Definition 2.5 We say that $G=\left(V, E,\left\{w_{j}\right\}\right)$ is a reduced instance of the line highway problem if $e_{j}=$ $\left[j_{s}, j_{t}\right] \subseteq V$ for each $e_{j} \in E$, where $V=[1, n]$ and $1 \leq j_{s} \leq j_{t} \leq n$.

Definition 2.6 We say that $G=\left(V, E,\left\{w_{j}\right\}\right)$ is a reduced instance of the cycle highway problem if $e_{j}=$ $\left[j_{s}, j_{t}\right] \subseteq V$ or $e_{j}=\left[j_{t}, n\right] \cup\left[1, j_{s}\right] \subseteq V$ for each $e_{j} \in E$, where $V=[1, n]$ and $1 \leq j_{s} \leq j_{t} \leq n$.

We say that $G=\left(V, E,\left\{w_{j}\right\}\right)$ is an instance of the line (or cycle) highway problem with $[s, \ell]$-valuation if $s=\min _{j \in[1, m]} w_{j}$ and $\ell=\max _{j \in[1, m]} w_{j}$. In particular, we say that $G=\left(V, E,\left\{w_{j}\right\}\right)$ is an instance of the line (or cycle) highway problem with a single valuation if $w_{j}=w>0$ for each $j \in[1, m]$.

\subsection{DAG Representation of the Line Highway Problem}

In this subsection, we present the DAG representation of the line highway problem due to Balcan, et al. [3, $\S 3]$. For a reduced instance $G=\left(V, E,\left\{w_{j}\right\}\right)$ of the line highway problem, define the DAG representation $H=\left(U, F,\left\{w_{j}\right\}\right)$ of $G$ as follows: For $V=\{1,2, \ldots, n\}$, let $U=\left\{u_{0}, u_{1}, \ldots, u_{n}\right\}$ be the set of $n+1$ vertices, and for each $e_{j}=\left[j_{s}, j_{t}\right] \in E$, let $f_{j}=\left(u_{j_{s}-1}, u_{j_{t}}\right) \in F$ be the arc $u_{j_{s}-1} \rightarrow u_{j_{t}}$ with weight $w_{j}$.

Let $\boldsymbol{p}=\left(p_{1}, p_{2}, \ldots, p_{n}\right)$ be a price vector for $G=\left(V, E,\left\{w_{j}\right\}\right)$. Then for the DAG representation $H=$ $\left(U, F,\left\{w_{j}\right\}\right)$ of $G$, define the partial sum for $u_{i} \in U$ by $s_{i}=\sum_{h=1}^{i} p_{h}$, where $s_{0}=0$. On the other hand, let $\boldsymbol{s}=\left(s_{0}, s_{1}, \ldots, s_{n}\right)$ be the partial sum vector for the DAG representation $H$ of $G$. Then we can define the price vector $\boldsymbol{p}=\left(p_{1}, p_{2}, \ldots, p_{n}\right)$ to be $p_{i}=s_{i}-s_{i-1}$ for each $i \in[1, n]$.

\section{Algorithms for the Line Highway Problem}

Balcan, et al. [3, Theorem 3] showed a 2.33-approximation algorithm for the line highway problem with a single valuation. In this section, we consider the line highway problem with $[s, \ell]$-valuation.

Let $G=\left(V, E,\left\{w_{j}\right\}\right)$ be a reduced instance of the line highway problem with $[s, \ell]$-valuation. For each $x \in[s, \ell]$, we use $E_{x}$ to denote the set of customers with valuation $x \in[s, \ell]$ and let $m_{x}=\left|E_{x}\right|$. Note that $m_{s}+m_{s+1}+\cdots+m_{\ell}=m=|E|$. Under the coupon model, let $\boldsymbol{p}_{\text {coup }}^{*}$ be the price vector with the maximum profit, and let $\operatorname{OPT}_{\text {coup }}(G)=\operatorname{PROFIT}_{\text {coup }}\left(\boldsymbol{p}_{\text {coup }}^{*}\right)$ be the maximum total profit returned from the customers in $E$. For each $x \in[s, \ell]$, we use $\operatorname{Opt}_{\text {coup }}^{x}(G)$ to denote the fraction of $\operatorname{OpT}_{\text {coup }}(G)$ that is returned from the customers in $E_{x}$ for the optimal price vector $\boldsymbol{p}_{\text {coup }}^{*}$. From the definition of $\operatorname{OpT}_{\text {coup }}^{x}(G)$ for each $x \in[s, \ell]$, we immediately have that $\operatorname{Opt}_{\text {coup }}(G)=\operatorname{OpT}_{\text {coup }}^{s}(G)+\operatorname{OpT}_{\text {coup }}^{s+1}(G)+\cdots+\operatorname{OpT}_{\text {coup }}^{\ell}(G)$.

Under the coupon model, our algorithm for the line highway problem with $[s, \ell]$-valuation consists of two algorithms LinE_RANDOM and Line_CuT.

\subsection{Algorithm: LINE_RANDOM}

In this subsection, we present the algorithm LinE_RANDOM for the line highway problem with $[s, \ell]$-valuation. The description of the algorithm LinE_RANDOM is given in Figure 1.

Theorem 3.1 On an instance $G=\left(V, E,\left\{w_{j}\right\}\right)$ of the line highway problem with $[s, \ell]$-valuation, the algorithm LiNE_RANDOM outputs a price vector $\boldsymbol{\sigma}$ that satisfies

$$
\frac{\operatorname{Opt}_{\text {coup }}(G)}{\mathbf{E}\left[\operatorname{PROFIT}_{\text {coup }}(\boldsymbol{\sigma})\right]} \leq\left\{\begin{array}{cl}
3 / r & 0<r \leq 1 / 2 \\
6 & 1 / 2<r \leq 1,
\end{array}\right.
$$


where $r=s / \ell$ is the ratio between the smallest and the largest valuations.

Input: A reduced instance $G=\left(V, E,\left\{w_{j}\right\}\right)$ of the line highway problem with $[s, \ell]$-valuation.

Output: A price vector $\boldsymbol{\sigma}=\left(\sigma_{1}, \sigma_{2}, \ldots, \sigma_{n}\right)$ for $G$.

1. Construct the DAG representation $H=\left(U, F,\left\{w_{j}\right\}\right)$ of $G=\left(V, E,\left\{w_{j}\right\}\right)$.

2. For each $u_{i} \in U$, assign a partial sum $s_{i} \in[0, \ell]$ for $u_{i}$ uniformly and at random.

3. For each $i \in V$, compute a price $\sigma_{i}=s_{i}-s_{i-1}$ for the item $i$ and let $\boldsymbol{\sigma}=\left(\sigma_{1}, \sigma_{2}, \ldots, \sigma_{n}\right)$.

Figure 1: The algorithm LinE_RANDOM

Proof: We begin by showing the following claims.

Claim 3.1 $\operatorname{Opt}_{\text {coup }}^{x}(G) \leq m_{x} \cdot x$ for each $x \in[s, \ell]$.

Proof: For each $x \in[s, \ell]$, the maximum profit returned from a customer $e_{j} \in E_{x}$ is at most $x$.

Claim 3.2 For each $x \in[s, \ell]$, let $\mathbf{E}\left[\operatorname{PROFIT}_{\text {coup }}^{x}(\boldsymbol{\sigma})\right]$ be the expected profit returned from the set $E_{x}$ of customers by the algorithm LINE_RANDOM. Then

$$
\mathbf{E}\left[\operatorname{PrOFIT}_{\text {coup }}^{x}(\boldsymbol{\sigma})\right]=\frac{m_{x}}{6(\ell+1)^{2}} \cdot x(x+1)(-2 x+3 \ell+2) .
$$

Proof: For each $x \in[s, \ell]$ and each $e \in E_{x}$, let $Y_{x}^{e}$ be the profit returned from a customer $e \in E_{x}$ in Step 2 of the algorithm Line_RAndom, and let $Y_{x}=\sum_{e \in E_{x}} Y_{x}^{e}$. For each $e \in E_{x}$, we estimate $\mathbf{E}\left[Y_{x}^{e}\right]$.

$$
\begin{aligned}
\mathbf{E}\left[Y_{x}^{e}\right] & =\frac{1}{(\ell+1)^{2}}\{1 \cdot \ell+2 \cdot(\ell-1)+\cdots+x \cdot(\ell-x+1)\} \\
& =\frac{1}{(\ell+1)^{2}} \sum_{k=1}^{x} k \cdot(\ell-k+1)=\frac{1}{6(\ell+1)^{2}} \cdot x(x+1)(-2 x+3 \ell+2) .
\end{aligned}
$$

Thus from the linearity of expectation [11] and the fact that $m_{x}=\left|E_{x}\right|$, it follows that for each $x \in[s, \ell]$, $\mathbf{E}\left[\operatorname{PrOFiT}_{\text {coup }}^{x}(\boldsymbol{\sigma})\right]=\mathbf{E}\left[Y_{x}\right]=\sum_{e \in E_{x}} \mathbf{E}\left[Y_{x}^{e}\right]=\left|E_{x}\right| \cdot \mathbf{E}\left[Y_{x}^{e}\right]=m_{x} \cdot \mathbf{E}\left[Y_{x}^{e}\right]$.

From Claims 3.1 and 3.2 , it follows that for each $x \in[s, \ell]$,

$$
\frac{\mathbf{E}\left[\operatorname{PrOFIT}_{\text {coup }}^{x}(\boldsymbol{\sigma})\right]}{\operatorname{OPT}_{\text {coup }}^{x}(G)} \geq \frac{1}{6(\ell+1)^{2}} \cdot(x+1)(-2 x+3 \ell+2) .
$$

Let $f(x)=(x+1)(-2 x+3 \ell+2)$ and let $F_{\min }=\min _{x \in[s, \ell]} f(x)$. Since the function $f$ is convex with respect to $x \in[s, \ell]$, we have that $F_{\min }=\min \{f(s), f(\ell)\}$. Let $g(s, \ell)=f(\ell)-f(s)=(\ell-2 s)(\ell-s)$ and this implies that $f(\ell) \geq f(s)$ if $\ell \geq 2 s ; f(\ell)<f(s)$ if $s \leq \ell<2 s$.

For the case that $\ell \geq 2 s$, it follows that $F_{\min }=f(s)$. So we have that for each $x \in[s, \ell]$,

$$
\begin{aligned}
\frac{\mathbf{E}\left[\operatorname{ProfIT}_{\text {coup }}^{x}(\boldsymbol{\sigma})\right]}{\operatorname{OPT}_{\text {coup }}^{x}(G)} & \geq \frac{f(x)}{6(\ell+1)^{2}} \geq \frac{f(s)}{6(\ell+1)^{2}}=\frac{(s+1)(3 \ell-2 s+2)}{6(\ell+1)^{2}} \\
& =\frac{1}{6} \cdot \frac{s+1}{\ell+1} \cdot \frac{3 \ell-2 s+2}{\ell+1} \geq \frac{1}{6} \cdot \frac{s}{\ell} \cdot\left(3-\frac{2 s+1}{\ell+1}\right) \\
& \geq \frac{1}{6} \cdot \frac{s}{\ell} \cdot\left(3-\frac{\ell+1}{\ell+1}\right)=\frac{1}{3} \cdot \frac{s}{\ell}=\frac{r}{3},
\end{aligned}
$$


which implies that $\mathbf{E}\left[\operatorname{PrOFIT}_{\text {coup }}^{x}(\boldsymbol{\sigma})\right] \geq(r / 3) \cdot \operatorname{OPT}_{\text {coup }}^{x}(G)$ for each $x \in[s, \ell]$. Thus we have that

$$
\begin{aligned}
& \frac{\mathbf{E}\left[\operatorname{Profit}_{\text {coup }}(\boldsymbol{\sigma})\right]}{\operatorname{OPT}_{\text {coup }}(G)}=\frac{\mathbf{E}\left[\operatorname{PrOFIT}_{\text {coup }}^{s}(\boldsymbol{\sigma})\right]+\mathbf{E}\left[\operatorname{PrOFIT}_{\text {coup }}^{s+1}(\boldsymbol{\sigma})\right]+\cdots+\mathbf{E}\left[\operatorname{PrOFIT}_{\text {coup }}^{\ell}(\boldsymbol{\sigma})\right]}{\operatorname{OPT}_{\text {coup }}^{s}(G)+\operatorname{OPT}_{\text {coup }}^{s+1}(G)+\cdots+\operatorname{OPT}_{\text {coup }}^{\ell}(G)} \\
& \geq \frac{r}{3} \cdot \frac{\mathrm{OPT}_{\text {coup }}^{s}(G)+\mathrm{OPT}_{\text {coup }}^{s+1}(G)+\cdots+\operatorname{OPT}_{\text {coup }}^{\ell}(G)}{\operatorname{OPT}_{\text {coup }}^{s}(G)+\operatorname{OPT}_{\text {coup }}^{s+1}(G)+\cdots+\operatorname{OPT}_{\text {coup }}^{\ell}(G)}=\frac{r}{3} .
\end{aligned}
$$

For the case that $s \leq \ell<2 s$, it follows that $F_{\min }=f(\ell)$. So we have that for each $x \in[s, \ell]$,

$$
\frac{\mathbf{E}\left[\operatorname{ProfiT}_{\mathrm{coup}}^{x}(\boldsymbol{\sigma})\right]}{\mathrm{OPT}_{\mathrm{coup}}^{x}(G)} \geq \frac{f(x)}{6(\ell+1)^{2}} \geq \frac{f(\ell)}{6(\ell+1)^{2}}=\frac{(\ell+1)(\ell+2)}{6(\ell+1)^{2}}=\frac{1}{6} \cdot \frac{\ell+2}{\ell+1}>\frac{1}{6},
$$

which implies that $\mathbf{E}\left[\operatorname{PrOFIT}_{\text {coup }}^{x}(\boldsymbol{\sigma})\right] \geq(1 / 6) \cdot \mathrm{OPT}_{\text {coup }}^{x}(G)$ for each $x \in[s, \ell]$. Thus in a way similar to the above, we have that $\mathbf{E}\left[\operatorname{ProfIT}_{\text {coup }}(\boldsymbol{\sigma}) \geq(1 / 6) \cdot \mathrm{OPT}_{\text {coup }}(G)\right.$.

\subsection{Algorithm: LINE_CUT}

In this subsection, we present the algorithm LinE_CUT for the line highway problem with $[s, \ell]$-valuation. The description of the algorithm Line_CUT is given in Figure 2.

Input: A reduced instance $G=\left(V, E,\left\{w_{j}\right\}\right)$ of the line highway problem with $[s, \ell]$-valuation.

Output: A price vector $\boldsymbol{\tau}=\left(\tau_{1}, \tau_{2}, \ldots, \tau_{n}\right)$ for $G$.

1. Construct the DAG representation $H=\left(U, F,\left\{w_{j}\right\}\right)$ of $G=\left(V, E,\left\{w_{j}\right\}\right)$.

2. Mark each $u \in U$ independently with probability $1 / 2$.

3. Let $L \subseteq U$ be the set of marked vertices and $R=U-L \subseteq U$ be the set of unmarked vertices.

4. Let $K$ be the set of arcs from the vertices in $L$ to the vertices in $R$.

5. For each $x \in[s, \ell]$, assign a partial sum 0 to all vertices $v \in L$ and a partial sum $x$ to all vertices $u \in$ $R$, and compute a price vector $\boldsymbol{\tau}_{x}$.

6. Output the price vector $\tau$ that satisfies

$$
\operatorname{PrOFIT}_{\text {coup }}(\boldsymbol{\tau})=\max _{x \in[s, \ell]} \operatorname{PROFIT}_{\text {coup }}\left(\boldsymbol{\tau}_{x}\right)
$$

Figure 2: The Algorithm LinE_CuT

Theorem 3.2 On an instance $G=\left(V, E,\left\{w_{j}\right\}\right)$ of the line highway problem with $[s, \ell]$-valuation, the algorithm LinE_CUT outputs a price vector $\boldsymbol{\tau}$ that satisfies

$$
\frac{\operatorname{Opt}_{\text {coup }}(G)}{\mathbf{E}\left[\operatorname{PrOFIT}_{\text {coup }}(\boldsymbol{\tau})\right]} \leq 4(1-\ln r)
$$

where $r=s / \ell$ is the ratio between the smallest and the largest valuations.

Proof: For the set $K$ of the arcs from the vertices in $L$ to the vertices in $R$, let $\operatorname{VAL}(K)$ be the sum of the valuations of the $\operatorname{arcs}$ in $K$. For each $x \in[s, \ell]$, let $K_{x}$ be the set of $\operatorname{arcs}$ in $K$ with valuation $x$ and let $m_{x}=$ $\left|K_{x}\right|$. Then it is immediate to see that $\operatorname{VAL}(K)=m_{s} \cdot s+m_{s+1} \cdot(s+1)+\cdots+m_{\ell} \cdot \ell$. To comlete the proof of the theorem, we need to show the following claims: 
Claim 3.3 $\mathbf{E}[\operatorname{VAL}(K)]=(1 / 4) \cdot \sum_{f_{j} \in F} w_{j}=(1 / 4) \cdot \sum_{e_{j} \in E} w_{j} \geq(1 / 4) \cdot \operatorname{OPT}_{\text {coup }}(G)$.

Proof: The first equality follows from the definition of $U$ and the first inequality is trivial.

Claim 3.4 For each $x \in[s, \ell]$, the following holds:

$$
\operatorname{VAL}(K)=\text { PrOFIT }_{\text {coup }}\left(\boldsymbol{\tau}_{s}\right)+\sum_{x=s+1}^{\ell} \frac{\operatorname{PrOFIT}_{\text {coup }}\left(\boldsymbol{\tau}_{x}\right)}{x} .
$$

Proof: From the definition of $\boldsymbol{\tau}_{x}$, we have that $\operatorname{PROFIT}_{\text {coup }}\left(\boldsymbol{\tau}_{x}\right)=m_{x} \cdot x+m_{x+1} \cdot x+\cdots+m_{\ell} \cdot x$ for each $x \in[s, \ell]$. Then the claim immediately follows from the definition of $\operatorname{VAL}(K)$.

From Claims 3.3 and 3.4 and the definition of $\tau$, it follows that

$$
\begin{aligned}
& \frac{1}{4} \cdot \operatorname{Opt}_{\text {coup }}(G) \leq \mathbf{E}[\operatorname{Vat}(K)]=\mathbf{E}\left[\operatorname{PrOFIT}_{\text {coup }}\left(\boldsymbol{\tau}_{s}\right)\right]+\sum_{x=s+1}^{\ell} \frac{\mathbf{E}\left[\operatorname{PrOFIT}_{\text {coup }}\left(\boldsymbol{\tau}_{x}\right)\right]}{x} \\
& \quad \leq \mathbf{E}\left[\operatorname{Profit}_{\text {coup }}(\boldsymbol{\tau})\right]+\sum_{x=s+1}^{\ell} \frac{\mathbf{E}\left[\operatorname{PrOFIT}_{\text {coup }}(\boldsymbol{\tau})\right]}{x}=\left(1+\sum_{k=s+1}^{\ell} \frac{1}{k}\right) \cdot \mathbf{E}\left[\operatorname{PROFIT} \operatorname{coup}_{\text {cou }}(\boldsymbol{\tau})\right] .
\end{aligned}
$$

Since $\sum_{k=s+1}^{\ell} 1 / k \leq \ln (\ell / s)=-\ln r$, we have that $\mathrm{OPT}_{\text {coup }}(G) \leq 4(1-\ln r) \cdot \mathbf{E}\left[\operatorname{PrOFIT}_{\text {coup }}(\boldsymbol{\tau})\right]$.

Remark 3.1 The algorithm LinE_CuT can be easily derandomized by applying pairwise independent 0/1random variables with a small sample space [12] in Step 2.

\subsection{Algorithm: $\operatorname{LINE}_{[s, \ell]}$}

The algorithm $\operatorname{LinE}_{[s, \ell]}$ works as follows: On an instance $G=\left(V, E,\left\{w_{j}\right\}\right)$ of the line highway problem with $[s, \ell]$-valuation, (1) run Line_RANDOM on $G$ to get the price vector $\boldsymbol{\sigma} ;(2)$ run Line_Cut on $G$ to get the price vector $\boldsymbol{\tau}$; (3) output the price vector $\boldsymbol{p}$ that satisfies

$$
\operatorname{Profit}_{\text {coup }}(\boldsymbol{p})=\max \left\{\operatorname{Profit}_{\text {coup }}(\boldsymbol{\sigma}), \operatorname{Profit}_{\text {coup }}(\boldsymbol{\tau})\right\} .
$$

From Theorems 3.1 and 3.2 , we immediately have the following theorem:

Theorem 3.3 On an instance $G=\left(V, E,\left\{w_{j}\right\}\right)$ of the line highway problem with $[s, \ell]$-valuation, the algorithm $\operatorname{LINE}_{[s, \ell]}$ outputs a price vector $\boldsymbol{p}$ that satisfies

$$
\frac{\operatorname{Opt}_{\text {coup }}(G)}{\mathbf{E}\left[\operatorname{ProfiT}_{\text {coup }}(\boldsymbol{p})\right]} \leq\left\{\begin{array}{cl}
4(1-\ln r) & 0 \leq r \leq \alpha, 1 / \sqrt{e} \leq r \leq 1 \\
3 / r & \alpha<r \leq 1 / 2 \\
6 & 1 / 2<r<1 / \sqrt{e}
\end{array}\right.
$$

where $r=s / \ell$ is the ratio between the smallest and the largest valuations and $\alpha \approx 0.3824$, i.e., $\alpha$ is the solution of the equality $3 / x=4(1-\ln x)$.

\section{Algorithms for the Cycle Highway Problem}

In this section, we first consider the cycle highway problems with $[s, \ell]$-valuation and then we consider the cycle highway problems with a single valuation as the special case of the cycle highway problem with $[s, \ell]$ valuation such that $s=\ell$, which also can be regarded as an extension of the line highway problem with a single valuation discussed by Balcan, et al. [3]. 


\subsection{Algorithms for the Cycle Highway Problem with $[s, \ell]$-Valuation}

In this subsection, we present an algorithm $\operatorname{CYCLE}_{[s, \ell]}$ for the cycle highway problem with $[s, \ell]$-valuation.

For a reduced instance $G=\left(V, E,\left\{w_{j}\right\}\right)$ of the cycle highway problem with $[s, \ell]$-valuation, define a directed graph $H=\left(U, F,\left\{w_{j}\right\}\right)$ as follows: Let $V=\{1,2, \ldots, n\}$ and each item $i \in V$ is arranged in a clockwise manner, i.e., we arrange $1 \rightarrow 2 \rightarrow \cdots \rightarrow n \rightarrow 1$. For each $e_{j} \in E$, let $1 \leq j_{s} \leq j_{t} \leq n$. If $e_{j}=\left[j_{s}, j_{t}\right] \subseteq$ $V$, then we define $f_{j}=\left(j_{s}, j_{t}\right)$ to be an arc $j_{s} \rightarrow j_{t}$ with valuation $w_{j}$; if $e_{j}=\left[j_{t}, n\right] \cup\left[1, j_{s}\right]$, then we define $f_{j}=\left(j_{t}, j_{s}\right)$ to be an arc $j_{t} \rightarrow j_{s}$ with valuation $w_{j}$. Let $F=\left\{f_{j}: e_{j} \in E\right\}$ be the set of arcs and let $U=\left\{j_{s} \in V: e_{j}=\left(j_{s}, j_{t}\right) \in E\right\} \cup\left\{j_{t} \in V: e_{j}=\left(j_{s}, j_{t}\right) \in E\right\}$ be the set of vertices.

The description of the algorithm $\mathrm{CYCLE}_{[s, \ell]}$ is given in Figure 3 ,

Input: A reduced instance $G=\left(V, E,\left\{w_{j}\right\}\right)$ of the cycle highway problem with $[s, \ell]$-valuation.

Output: A price vector $\boldsymbol{p}=\left(p_{1}, p_{2}, \ldots, p_{n}\right)$ for $G$.

1. For $G=\left(V, E,\left\{w_{j}\right\}\right)$, construct a directed graph $H=\left(U, F,\left\{w_{j}\right\}\right)$.

2. Mark each $u \in U$ independently with probability $1 / 2$.

3. Let $L \subseteq U$ be the set of marked vertices and $R=U-L \subseteq U$ be the set of unmarked vertices.

4. Let $J_{H}=\left\{f_{j}=\left(a_{j}, b_{j}\right) \in F: a_{j} \in L, b_{j} \in R\right\}$ be the set of arcs from the vertices in $L$ to the vertices in $R$ and remove all arcs in $F-J_{H}$.

5. For each $x \in[s, \ell]$, assign a partial sum $-x / 2$ to all vertices $v \in L$ and a partial sum $x / 2$ to all vertices $u \in R$, and compute a price vector $\boldsymbol{p}_{x}$.

6. Output the price vector $\boldsymbol{p}$ that satisfies $\operatorname{ProfiT}_{\text {coup }}(\boldsymbol{p})=\max _{x \in[s, \ell]} \operatorname{PROFIT}_{\text {coup }}\left(\boldsymbol{p}_{x}\right)$.

Figure 3: The Algorithm CYCLE $[s, \ell]$

Theorem 4.1 On an instance $G=\left(V, E,\left\{w_{j}\right\}\right)$ of the cycle highway problem with $[s, \ell]$-valuation, the algorithm $\mathrm{CYCLE}_{[s, \ell]}$ outputs a price vector $\boldsymbol{p}$ that satisfies

$$
\operatorname{OPT}_{\text {coup }}(G) \leq 4(1-\ln r) \cdot \mathbf{E}[\operatorname{PrOFIT} \text { coup }(\boldsymbol{p})],
$$

where $r=s / \ell$ is the ratio between the smallest and the largest valuations.

Proof: For the set $J_{H}$ of arcs from the vertices in $L$ to the vertices in $R$, let $\operatorname{VAL}\left(J_{H}\right)$ be the sum of the valuations of the $\operatorname{arcs}$ in $J_{H}$. For each $x \in[s, \ell]$, let $J_{H}^{x}$ be the set of $\operatorname{arcs}$ in $J_{H}$ with valuation $x$ and let $m_{x}=$ $\left|J_{H}^{x}\right|$. Then we can show the following claims:

Claim $4.1 \mathbf{E}\left[\operatorname{VAL}\left(J_{H}\right)\right]=(1 / 4) \cdot \sum_{f_{j} \in F} w_{j}=(1 / 4) \cdot \sum_{e_{j} \in E} w_{j} \geq(1 / 4) \cdot \operatorname{OPT}_{\text {coup }}(G)$.

Proof: This can be shown in a way similar to the proof of Claim 3.3 ,

Claim 4.2 For the price vector $\boldsymbol{p}_{s}, \boldsymbol{p}_{s+1}, \ldots, \boldsymbol{p}_{\ell}$ and $\operatorname{VAL}\left(J_{H}\right)$, the following holds:

$$
\operatorname{VAL}\left(J_{H}\right)=\text { PROFIT }_{\text {coup }}\left(\boldsymbol{p}_{s}\right)+\sum_{x=s+1}^{\ell} \frac{\operatorname{PROFIT}_{\text {coup }}\left(\boldsymbol{p}_{x}\right)}{x} .
$$

Proof: Note that each vertex $v \in L$ has no incoming arcs and each vertex $u \in R$ has no outgoing arcs. Define the set $J_{G}$ of intervals to be $e_{j} \in J_{G}$ if $f_{j} \in J_{H}$. Thus for each $x \in[s, \ell]$, assigning a partial sum $-x / 2$ to all vertices $v \in L$ and assigning a partial sum $x / 2$ to all vertices $u \in R$ implies that each $e_{j} \in J_{G}$ is assigned 
$x=x / 2-(-x / 2)$ as a total sum of prices for the corresponding items in $e_{j}$. To define a price vector $\boldsymbol{p}_{x}$, we appropriately assign prices to all items that are not assigned prices (this does not reduce the profit returned from the customers $\left.e_{j} \in J_{G}\right)$. Then we have that $\operatorname{PROFIT}_{\text {coup }}\left(\boldsymbol{p}_{x}\right)=m_{x} \cdot x+m_{x+1} \cdot x+\cdots+m_{\ell} \cdot x$ for each $x \in[s, \ell]$. In a way similar to the proof of Claim 3.4, the claim follows from the definition of $\operatorname{VAL}\left(J_{H}\right)$, i.e., $\operatorname{VAL}\left(J_{H}\right)=m_{s} \cdot s+m_{s+1} \cdot(s+1)+\cdots+m_{\ell} \cdot \ell$.

From Claims 4.1 and 4.2 and the definition of $\boldsymbol{p}$, it follows that

$$
\begin{aligned}
& \frac{1}{4} \cdot \operatorname{Opt}_{\text {coup }}(G) \leq \mathbf{E}\left[\operatorname{VaL}\left(J_{H}\right)\right]=\mathbf{E}\left[\operatorname{PrOFIT}_{\text {coup }}\left(\boldsymbol{p}_{s}\right)\right]+\sum_{x=s+1}^{\ell} \frac{\mathbf{E}\left[\operatorname{PrOFIT}_{\text {coup }}\left(\boldsymbol{p}_{x}\right)\right]}{x} \\
& \quad \leq \mathbf{E}\left[\operatorname{Profit}_{\text {coup }}(\boldsymbol{p})\right]+\sum_{x=s+1}^{\ell} \frac{\mathbf{E}\left[\operatorname{PrOFIT}_{\text {coup }}(\boldsymbol{p})\right]}{x}=\left(1+\sum_{k=s+1}^{\ell} \frac{1}{k}\right) \cdot \mathbf{E}\left[\operatorname{PROFIT}_{\text {coup }}(\boldsymbol{p})\right] .
\end{aligned}
$$

Since $\sum_{k=s+1}^{\ell} 1 / k \leq \ln (\ell / s)=-\ln r$, we have that $\operatorname{OPT}_{\text {coup }}(G) \leq 4(1-\ln r) \cdot \mathbf{E}[\operatorname{PrOFIT}$ coup $(\boldsymbol{p})]$.

Remark 4.1 The algorithm $\mathrm{CYCLE}_{[s, \ell]}$ can be easily derandomized by applying pairwise independent 0/1random variables with a small sample space [12] in Step 2.

\subsection{Algorithms for the Cycle Highway Problem with a Single Valuation}

Let $G=\left(V, E,\left\{w_{j}\right\}\right)$ be a reduced instance of the cycle highway problem with a single valuation, i.e., $r=1$. So it follows from Theorem 4.1 that $\operatorname{OPT}_{\text {coup }}(G) \leq 4 \cdot \operatorname{PROFIT}_{\text {coup }}(\boldsymbol{p})$. To improve this, we present the algorithm CYC_SingLE_VAL for the cycle highway problem with a single valuation. Without loss of generality, we assume that $w_{j}=1$ for each customer $e_{j} \in E$ and we use $G=(V, E)$ to denote an instance of the cycle highway problem with a single valuation. The algorithm CyC_SInGLE_VAL is given in Figure 4.

Theorem 4.2 On an instance $G=(V, E)$ of the cycle highway problem with a single valuation, the algorithm CYC_SINGLE_VAL outputs a price vector $\boldsymbol{p}$ that satisfies

$$
\operatorname{OPT}_{\text {coup }}(G) \leq 2.747 \cdot \operatorname{PrOFIT}_{\text {coup }}(\boldsymbol{p}) \text {. }
$$

Proof: As in Subsection 4.2, it is obvious that $E_{\text {in }} \cup E_{\text {out }} \subseteq V$. Without loss of generality, we assume that $E_{\text {in }} \cup E_{\text {out }}=V$ (otherwise the instance $G$ of the cycle highway problem with a single valuation can be regarded as an instance of the line highway problem with a single valuation, which has a 2.33-approximation algorithm due to Balcan, et al [3, Theorem 3]). Let $\boldsymbol{p}_{\text {coup }}^{*}$ be the price vector with the maximum profit and $\operatorname{OPT}_{\text {coup }}(G)=\operatorname{PROFIT}_{\text {coup }}\left(\boldsymbol{p}_{\text {coup }}^{*}\right)$ be the maximum profit returned from the customers in $E$. For the optimal price vector $\boldsymbol{p}_{\text {coup }}^{*}$, we use $\mathrm{OPT}_{\text {coup }}^{\text {in }}(G)$ to denote the fraction of $\operatorname{OpT}_{\text {coup }}(G)$ that are returned from the customers in $E_{\text {in }}$, and we also use $\operatorname{OpT}_{\text {coup }}^{\text {out }}(G)$ to denote the fraction of $\operatorname{OpT}_{\text {coup }}(G)$ that are returned from the customers in $E_{\text {out }}$. It is obvious that $\operatorname{OpT}_{\text {coup }}(G)=\operatorname{OpT}_{\text {coup }}^{\text {in }}(G)+\operatorname{OpT}_{\text {coup }}^{\text {out }}(G)$.

For each $e_{j} \in E_{\text {in }}$, let $e_{j}^{L} \subseteq e_{j}-\{h\}$ (resp. $e_{j}^{R} \subseteq e_{j}-\{h\}$ ) be the subinterval on the left (resp. the right) of $h$. For each $e_{j} \in E_{\text {in }}$, we have that $e_{j}^{L} \cap e_{j}^{R}=\emptyset$ and $e_{j}=e_{j}^{L} \cup e_{j}^{R} \cup\{h\}$. To complete the proof of the theorem, we need to show the following claims:

Claim 4.3 $\operatorname{Profit}_{\text {coup }}(\boldsymbol{\sigma})=\left|E_{\text {in }}\right| \geq \operatorname{Opt}_{\text {coup }}^{\text {in }}(G)$.

Proof: This follows from the fact that each customer in $E_{\text {in }}$ provides profit "1."

Claim 4.4 $\operatorname{PROFIT}_{\text {coup }}\left(\boldsymbol{\tau}_{\text {out }}\right) \geq \operatorname{OPT}_{\text {coup }}^{\text {out }}(G) / a$, where $a \approx 2.33$.

Proof: This follows from the result due to Balcan, et al. [3, Theorem 3]. 
Input: A reduced instance $G=(V, E)$ of the cycle highway problem with a single valuation. Output: A price vector $\boldsymbol{p}=\left(p_{1}, p_{2}, \ldots, p_{n}\right)$ for $G$.

1. Choose an item $h \in V$ arbitrarily.

2. Let $J_{\text {in }}=\left\{j \in[1, m]: e_{j} \in E, h \in e_{j}\right\}$ and $E_{\text {in }}=\left\{e_{j} \in E: i \in J_{\text {in }}\right\}$. Let $V_{\text {in }}=\cup_{j \in J_{\text {in }}} e_{j}$ be the set of items that the customers in $E_{\text {in }}$ are interested in.

3. Let $J_{\text {out }}=\left\{j \in[1, m]: e_{j} \in E, h \notin e_{j}\right\}$ and $E_{\text {out }}=\left\{e_{j} \in E: j \in J_{\text {out }}\right\}$. Let $V_{\text {out }}=\cup_{j \in J_{\text {out }}} e_{j}$ be the set of items that the customers in $E_{\text {out }}$ are interested in.

4. Define a price vector $\boldsymbol{\sigma}$ by assigning $x$ to 1 and by assigning 0 to all $i \in V-\{h\}$.

5. Regard $G_{\text {out }}=\left(V_{\text {out }}, E_{\text {out }}\right)$ as an instance of the line highway problem with a single valuation.

6. On input $G_{\text {out }}=\left(V_{\text {out }}, E_{\text {out }}\right)$, run the algorithm due to Balcan, et al. [3, Theorem 3] to compute a price vector $\tau_{\text {out }}$ for the set $V_{\text {out }}$ of items.

7. For each $x \in\{-1,0,1,2\}$, define a price vector $\boldsymbol{\tau}_{\text {in }}^{x}$ for the set $V_{\text {in }}$ of items by assigning $x$ to $h$ and by assigning 0 to all $i \in V_{\text {in }}-\left(V_{\text {out }} \cup\{h\}\right)$, and let $\boldsymbol{\tau}=\left(\boldsymbol{\tau}_{\text {out }}, \boldsymbol{\tau}_{\text {in }}\right)$, where

$$
\operatorname{PROFIT}_{\text {coup }}\left(\boldsymbol{\tau}_{\text {in }}\right)=\max _{x \in\{-1,0,1,2\}} \operatorname{PROFIT}_{\text {coup }}\left(\boldsymbol{\tau}_{\text {in }}^{x}\right) .
$$

8. Output the price vector $\boldsymbol{p}$ that satisfies

$$
\operatorname{PrOFIT}_{\text {coup }}(\boldsymbol{p})=\max \left\{\operatorname{PrOFIT}_{\text {coup }}(\boldsymbol{\sigma}), \operatorname{PROFIT}_{\text {coup }}(\boldsymbol{\tau})\right\}
$$

Figure 4: The Algorithm CyC_Single_VAL

Claim 4.5 For each $e_{j} \in E_{\mathrm{in}}$, the sum of the prices for the items in $e_{j}^{R}$ is either 0 or 1 , and the sum of the prices for the items in $e_{j}^{L}$ is either $-1,0$, or 1 .

Proof: For $G_{\text {out }}=\left(V_{\text {out }}, E_{\text {out }}\right)$ in Step 6 of the algorithm CyC_Single_VAL, let $H_{\text {out }}=\left(U_{\text {out }}, F_{\text {out }}\right)$ be the DAG representation of $G_{\text {out }}$ and let $U_{\text {out }}=\left\{u_{0}, u_{1}, \ldots, u_{k}\right\}$. Let $s=\left(s_{0}, s_{1}, \ldots, s_{k}\right)$ be the partial sum vector output by the algorithm due to Balcan, et al. [3, Theorem 3] on $H_{\text {out }}$. On input $H_{\text {out }}$, the algorithm [3, Theorem 3] computes the directed cut $\left(U_{\text {out }}^{L}: U_{\text {out }}^{R}\right)$ by running the algorithm due to Feige and Goemans [8] and defines the partial sum vector $s$ by assigning 0 to all $i \in U_{\text {out }}^{L}$ and by assigning 1 to al $i \in U_{\text {out }}^{R}$. For the DAG representation $H_{\text {out }}=\left(U_{\text {out }}, F_{\text {out }}\right)$ of $G_{\text {out }}$, it is easy to see that $u_{0} \in U_{\text {out }}$ has no incoming arcs. Thus if $u_{0} \in U_{\text {out }}^{R}$, then by moving $u_{0}$ from $U_{\text {out }}^{R}$ to $U_{\text {out }}^{L}$, we have the $\left.\operatorname{directed~cut~}\left(U_{\text {out }}^{L} \cup\left\{u_{0}\right\}: U_{\text {out }}^{R}-\left\{u_{0}\right\}\right\}\right)$ including more crossing arcs than the directed cut $\left.\left(U_{\text {out }}^{L}: U_{\text {out }}^{R}\right\}\right)$. So without loss of generality, we assume that $u_{0} \in U_{\text {out }}^{L}$ for the directed cut $\left(U_{\text {out }}^{L}, U_{\text {out }}^{R}\right)$. This implies that $s_{0}=0$ and $s_{i} \in\{0,1\}$ for each $1 \leq i \leq k$.

For each $e_{j} \in E_{\text {in }} \subseteq V_{\text {in }}$, let $e_{j}^{\prime}=e_{j} \cap V_{\text {out }}=\left\{v_{1}^{\text {out }}, v_{2}^{\text {out }}, \ldots, v_{t}^{\text {out }}\right\}$. So the sum of the prices for the items $v_{1}^{\text {out }}, v_{2}^{\text {out }}, \ldots, v_{t}^{\text {out }}$ is $s_{t}-s_{0}=s_{t} \in\{0,1\}$. On the other hand, we have assigned 0 to all $i \in V_{\text {in }}-\left(V_{\text {out }} \cup\{h\}\right)$ in Step 7 of the algorithm CyC_Single_VAL, which implies that for each $e_{j} \in E_{\text {in }}$, the sum of the prices for the items $i \in e_{j}-\left(V_{\text {out }} \cup\{h\}\right)$ is 0 . Thus we have that for each $e_{j} \in E_{\text {in }}$, the sum of the prices for the items in $e_{j}^{R}$ is $s_{t} \in\{0,1\}$. In a way similar to $e_{j}^{R}$, we can immediately show that for each $e_{j} \in E_{\text {in }}$, the sum of the prices for the items in $e_{j}^{L}$ is either $-1,0$, or 1 .

Claim 4.6 $\operatorname{Profit}_{\text {coup }}(\tau) \geq \operatorname{OPT}_{\text {coup }}^{\text {out }}(G) / a+\operatorname{OPT}_{\text {coup }}^{\text {in }}(G) / 4$, where $a \approx 2.33$.

Proof: Define $\mathcal{M}_{h}, \mathcal{M}_{L}, \mathcal{M}_{R}, \mathcal{M}_{L R} \subseteq E_{\text {in }}$ as follows:

$$
\mathcal{M}_{h}=\left\{e_{j} \in E_{\text {in }}: e_{j}^{L}=e_{j}^{R}=\emptyset\right\}
$$




$$
\begin{aligned}
\mathcal{M}_{L} & =\left\{e_{j} \in E_{\text {in }}: e_{j}^{L} \neq \emptyset, e_{j}^{R}=\emptyset\right\} ; \\
\mathcal{M}_{R} & =\left\{e_{j} \in E_{\text {in }}: e_{j}^{L}=\emptyset, e_{j}^{R} \neq \emptyset\right\} ; \\
\mathcal{M}_{L R} & =\left\{e_{j} \in E_{\text {in }}: e_{j}^{L} \neq \emptyset, e_{j}^{R} \neq \emptyset\right\} .
\end{aligned}
$$

From Claim 4.5, we have that for each $e_{j} \in E_{\text {in }}$, the sum of the prices for the items in $e_{j}^{R}$ is either 0 or 1 , and the sum of the prices for the items in $e_{j}^{L}$ is either $-1,0$, or 1 . For each $\beta \in\{-1,0,1\}$ and each $\gamma \in\{0,1\}$, we further partition $\mathcal{M}_{L}, \mathcal{M}_{R}$, and $\mathcal{M}_{L R}$ according to the sum of the prices.

$$
\begin{aligned}
\mathcal{M}_{L}^{(\beta)} & =\left\{e_{j} \in \mathcal{M}_{L}: \text { the sum of the prices of items in } e_{j}^{L} \text { is } \beta\right\} \\
\mathcal{M}_{R}^{(\gamma)} & =\left\{e_{j} \in \mathcal{M}_{R}: \text { the sum of the prices of items in } e_{j}^{R} \text { is } \gamma\right\} \\
\mathcal{M}_{L R}^{(\beta, \gamma)} & =\left\{e_{j} \in \mathcal{M}_{L R}: \text { the sum of the prices of items in } e_{j}^{L} \text { is } \beta\right. \\
& \text { and the sum of the prices of items in } \left.e_{j}^{R} \text { is } \gamma\right\} .
\end{aligned}
$$

If the price of $h \in V$ is $x \in\{-1,0,1,2\}$, then from the customers in $E_{\mathrm{in}}$, we can get

$$
\operatorname{PrOFit}_{\text {coup }}\left(\boldsymbol{\tau}_{\text {in }}^{x}\right)= \begin{cases}\left|\mathcal{M}_{L R}^{(1,1)}\right| & x=-1 \\ \left|\mathcal{M}_{L}^{(1)}\right|+\left|\mathcal{M}_{R}^{(1)}\right|+\left|\mathcal{M}_{L R}^{(0,1)}\right|+\left|\mathcal{M}_{L R}^{(1,0)}\right| & x=0 \\ \left|\mathcal{M}_{h}\right|+\left|\mathcal{M}_{L}^{(0)}\right|+\left|\mathcal{M}_{R}^{(0)}\right|+\left|\mathcal{M}_{L R}^{(-1,1)}\right|+\left|\mathcal{M}_{L R}^{(0,0)}\right| & x=1 \\ \left|\mathcal{M}_{L}^{(-1)}\right|+\left|\mathcal{M}_{L R}^{(-1,0)}\right| & x=2\end{cases}
$$

Thus in Step 7, we have that $\operatorname{PrOFIT}_{\text {coup }}\left(\boldsymbol{\tau}_{\text {in }}\right) \geq\left|E_{\text {in }}\right| / 4 \geq \operatorname{OPT}_{\text {coup }}^{\text {in }}(G) / 4$, and it follows from Claim 4.4 that $\operatorname{PrOFIT}_{\text {coup }}(\boldsymbol{\tau}) \geq \operatorname{OPT}_{\text {coup }}^{\text {out }}(G) / a+\operatorname{OPT}_{\text {coup }}^{\text {in }}(G) / 4$, where $a \approx 2.33$.

From Claims 4.3 and 4.6, we have that

$$
\begin{aligned}
\operatorname{Profit}_{\text {coup }}(\boldsymbol{p}) & =\max \left\{\operatorname{PrOFIT}_{\text {coup }}(\boldsymbol{\sigma}), \operatorname{PrOFIT}_{\text {coup }}(\boldsymbol{\tau})\right\} \\
& \geq \max \left\{\operatorname{OPT}_{\text {coup }}^{\text {in }}(G), \frac{\mathrm{OPT}_{\text {coup }}^{\text {out }}(G)}{a}+\frac{\mathrm{OPT}_{\text {coup }}^{\text {in }}(G)}{4}\right\} \\
& \geq \frac{4-a}{3 a+4} \cdot \operatorname{OPT}_{\text {coup }}^{\text {in }}(G)+\frac{4 a}{3 a+4} \cdot\left\{\frac{\operatorname{OPT}_{\text {coup }}^{\text {out }}(G)}{a}+\frac{\operatorname{OPT}_{\text {coup }}^{\text {in }}(G)}{4}\right\} \\
& =\frac{4}{3 a+4} \cdot\left\{\operatorname{OPT}_{\text {coup }}^{\text {in }}(G)+\operatorname{OPT}_{\text {coup }}^{\text {out }}(G)\right\}=\frac{4}{3 a+4} \cdot \operatorname{OPT}_{\text {coup }}(G) .
\end{aligned}
$$

Since $a \approx 2.33$, we have that the algorithm CYC_Single_VAL is a 2.747-approximation algorithm for the cycle highway problem with a single valuation.

\section{Concluding Remarks}

In this paper, we have considered the line and cycle highway problems with $[s, \ell]$-valuation or a single valuation and have shown their approximation algorithms.

Balcan, et al. [2, Theorem 15] consider the tree highway problem with a single valuation as a natural extension of the line highway problem with a single valuation and showed that it has a 4-approximation algorithm. As a straightforward extension of Theorem 3.1, we can easily show the following theorem for the tree highway problem with $[s, \ell]$-valuation. 
Input: A reduced instance $G=\left(V, E,\left\{w_{j}\right\}\right)$ of the tree highway problem with $[s, \ell]$-valuation.

Output: A price vector $\boldsymbol{\sigma}=\left(\sigma_{1}, \sigma_{2}, \ldots, \sigma_{n}\right)$ for $G$.

1. Choose $r \in V$ arbitrarily as a root and construct the DAG representation $H=\left(U, F,\left\{w_{j}\right\}\right)$ of $G=\left(V, E,\left\{w_{j}\right\}\right)$.

2. For each $u_{i} \in U$, assign a partial sum $s_{i} \in[0, \ell]$ for $u_{i}$ uniformly and at random.

3. For each $i \in V$, compute a price $\sigma_{i}=s_{i}-s_{i-1}$ for the item $i$ and let $\boldsymbol{\sigma}=\left(\sigma_{1}, \sigma_{2}, \ldots, \sigma_{n}\right)$.

Figure 5: The algorithm LinE_RANDOM

Theorem 5.1 On an instance $G=\left(V, E,\left\{w_{j}\right\}\right)$ of the tree highway problem with $[s, \ell]$-valuation, the algorithm TREE_RANDOM outputs a price vector $\boldsymbol{\sigma}$ that satisfies

$$
\operatorname{OpT}_{\text {coup }}(G) \leq \frac{16}{3 r} \cdot \mathbf{E}[\operatorname{ProfiT} \text { coup }(\boldsymbol{\sigma})]
$$

where $r=s / \ell$ is the ratio between the smallest and the largest valuations.

In this paper, we have focused on the highway problem under the coupon model, however, we do not know much about the general item pricing problem under the other models such as the discount model, the $B$-bounded discount model, etc. So the interesting problem to be considered is

(1) Design algorithms for the general item pricing problems under the ( $B$-bounded) discount model.

We are also interested in the inapproximability for the line and cycle highway problems with $[s, \ell]$-valuation or a single valuation. Thus the interesting problem to be considered is

(2) Derive the nontrivial lower bounds on the approximability for the line and cycle highway problems with $[s, \ell]$-valuation or a single valuation.

\section{References}

[1] M.F. Balcan and A. Blum. Approximation Algorithms and Online Mechanisms for Item Pricing. In Proc. of the 7th ACM Conference on Electronic Commerce, 29-35, 2006.

[2] M.F. Balcan, A. Blum, T-H. H. Chan, and M. Hajiaghayi. A Theory of Loss Leaders: Making Money by Pricing Below Cost. School of Computer Science, Carnegie Mellon University, Technical Report, CMU-CS-07-142, 2007.

[3] M.F. Balcan, A. Blum, T-H. H. Chan, and M. Hajiaghayi. A Theory of Loss Leaders: Making Money by Pricing Below Cost. In Proc. of the 3rd International Workshop on Internet and Network Economics, Lecture Notes in Computer Science 4858, 293-299, 2007.

[4] P. Briest and P. Krysta. Single-Minded Unlimited Supply Pricing on Sparce Instances. In Proc. of the 17th Annual ACM-SIAM Symposium on Discrete Algorithms, 1093-1102, 2006.

[5] P. Briest and P. Krysta. Buying Cheap is Expensive: Hardness of Non-Parametric Multi-Product Pricing. In Proc. of the 18th Annual ACM-SIAM Symposium on Discrete Algorithms, 716-725, 2007.

[6] P. DeGraba. Volume Discounts, Loss Leaders, and Competition for More Profitable Customers. Federal Trade Commission Bureau of Economics, Working Paper 260. 2003. 
[7] E.D. Demaine, U. Feige, M. Hajiaghayi, and M.R. Salavatipour. Combination Can Be Hard: Approximability of the Unique Coverage Problem. In Proc. of the 17th Annual ACM-SIAM Symposium on Discrete Algorithms, 162-171, 2006.

[8] U. Feige and M.X. Goemans. Approximating the Value of Two Prover Proof Systems, with Applications to MAX 2SAT and MAX DICUT. In Proc. $f$ the 3rd Israel Symposium on the Theory of Computing Systems, 182-189, 1995.

[9] A. Grigoriev, J. van Loon, R. Sitters, and M. Uetz. How to Sell a Graph: Guidelines for Graph Retailers. In Proc. of the 32nd International Workshop on Graph-Theoretic Concepts in Computer Science, Lecture Notes in Computer Science 4271, 125-136, 2006.

[10] V. Guruswami, J.D. Hartline, A.R. Karlin, D. Karger, C. Kenyon, and F. McSherry. On ProfitMaximizing Envy-Free Pricing. In Proc. of the 16th Annual ACM-SIAM Symposium on Discrete Algorithms, 1164-1174, 2005.

[11] R. Motowani and P. Raghavan. Randomized Algorithms. Cambridge University Press, 1995.

[12] M. Luby and A. Wigderson. Pairwise Independence and Derandomization. Technical Report: CSD95-880, 1995. 